\title{
Cytotoxicity of three-dimensional paper-based models from a three-dimensional paper-based printer
}

\author{
Authors: \\ Kozakiewicz M (DDS, PhD, Prof) ${ }^{1, *}$, \\ Szymor $P(M D, D M D)^{1}$ \\ Olszewski R (DDS, MD, PhD, Prof)
}

\begin{abstract}
Affiliations:
${ }^{1}$ Department of Maxillofacial Surgery, Medical University of Lodz, ul. Zeromskiego 113, 90-549 Lodz, Poland

${ }^{2}$ Department of oral and maxillofacial surgery, Cliniques universitaires saint Luc, Université catholique de Louvain, Av. Hippocrate 10, 1200 Brussels, Belgium, *Corresponding author: Kozakiewicz M, Department of maxillofacial surgery, , Medical University of Lodz, ul. Zeromskiego 113, 90-549 Lodz, Poland

Email: marcin.kozakiewicz@umed.lodz.pl

ORCID iD: 0000-0001-9200-2828

Disclaimer: the views expressed in the submitted article are our own and not an official position of the institution or funder.
\end{abstract}




\title{
Cover letter
}

24

\author{
Dear Editor-in-Chief,
}

Please receive our article titled "Sterilization methods and cytotoxicity of threedimensional paper-based models from a three-dimensional paper-based printer" for open evaluation in Nemesis journal.

1) Summarize the study's contribution to the scientific literature: Our study aimed to determine the possibility of using 3D models created with a low-cost, paper-based $3 \mathrm{D}$ printer in an operating room. Therefore, the influence of different methods of sterilization on $3 \mathrm{D}$ models was tested, and the cytotoxicity of generated $3 \mathrm{D}$ models was also determined.

2) Relate the study to previously published work: there was no previous works on sterilization methods and cytotoxicity evaluation of three-dimensional paper-based models generated using three-dimensional printer Mcor (Mcor technology, Eire).

3) Specify the type of article (for example, research article, systematic review, meta-analysis, clinical trial): we provide with experimental research article.

4) Describe any prior interactions with Nemesis regarding the submitted manuscript: we have no prior interactions with Nemesis journal.

5) Nemesis aim and scope relevance: There was no statistically significant difference for established statistical significance $\mathrm{p}=0.05$ in cuboids dimensions before and after sterilization regardless of sterilization method. For cytotoxicity, all 3D paperprinted and sterilized samples showed higher cytotoxicity against normal, human, adult dermal fibroblast culture when compared to positive control. The ANOVA statistical analysis confirmed that only 2-octyl cyanoacrylate coating of $3 \mathrm{D}$ paper model improved the biological behaviour of the material. 
Abstract

Objective: Our study aimed to determine the possibility of using models created with a low-cost, paper based 3D printer in an operating room. Therefore influence of different methods of sterilization on models was tested and cytotoxicity of generated models was determined.

Material and methods: 30 cuboids divided into three groups were used for verification of shape stability after sterilization. Each group was sterilized either with: Ethylene oxide in temperature $55^{\circ} \mathrm{C}$, Hydrogen peroxide gas plasma in temperature $60^{\circ} \mathrm{C}$ or Gamma irradiation at $21^{\circ} \mathrm{C}, 25 \mathrm{kGy}$. Each cuboid was measured using calliper three times before and three times after sterilization. Results were analysed statistically in Statgraphics Plus. Statistical significance was determined as $\mathrm{p}<0.05$. Sixty cylinders divided into six groups were used for cytotoxicity tests. Three of those groups were covered before sterilization with 2-octyl-cyanoacrylate. Each group was sterilized with one of the previously described methods. Cytotoxicity was tested by Nanostructural and Molecular Biophysics Laboratory in Technopark Lodz using normal adult human dermal fibroblasts. Survival of cells was tested using spectrophotometry with XTT and was defined as ratio of absorbency of tested probe to absorbency of control probe. Calcein/Ethidium dyeing test was performed according to LIVE/DEAD Viability/Cytotoxicity Kit protocol. Observation was done under Olympus GX71 fluorescence microscope. Results: There was no statistically significant difference for established statistical significance $\mathrm{p}=0.05$ in cuboids dimensions before and after sterilization regardless of sterilization method. In XTT analysis all samples showed higher cytotoxicity against normal, human, adult dermal fibroblast culture when compared to positive control. ANOVA statistical analysis confirmed that 2-octyl cyanoacrylate coating of paper model improved biological behaviour of the material. It decreased cytotoxicity of the model independently of sterilization method. In calcein/ethidium dyeing test due to the high fluorescence of the background caused by cylinders of analysed substance it was impossible to perform the exact analysis of the number of marked cells.

Conclusions: Acquired results allow to conclude that Mcor Technology Matrix $3003 \mathrm{D}$ paper-based models can be used in operating room only if covered with cyanoacrylate tissue adhesive.

Nemesis relevance: no statistically significant difference in cuboids dimensions before and after sterilization regardless of sterilization method. Presence of high cytotoxicity of 3D paper-based models without coating.

Keywords:

cytotoxicity; sterilization; three-dimensional printing, three-dimensional printer 
[Nemesis] Cytotoxicity of three-dimensional paper-based models from a three-dimensional paper-based printer

\section{Introduction}

Within last few years patient-customized craniofacial reconstructions become new standard in maxillofacial surgery. Patient specific 3D printed models can be used as a template for pre-shaping titanium mesh [1-6] or bone grafts $[1,7,8]$ before implantation, to simulate osteotomies $[1,9,10]$, to improve cancer resection techniques $[11,12]$ and to better plan the surgery [7]. There are even possibilities to produce patient-specific implants using either subtractive $[2,13]$ or additive methods like selective laser sintering [6,14-17]. All of these innovations lead to reduce operating time, minimize complications and produce better fitted implants [2], and improve functional and aesthetic postoperative results [2, 17].

The most commonly $3 \mathrm{D}$ printing technique in maxillofacial surgery is stereolithography using liquid resin which is photopolymerised with a laser beam [6]. Models created with this material have already proven their usefulness in medicine $[1,4,14$, 17-21]. Three-dimensional objects manufactured in such 3D printer are durable enough to successfully undergo sterilization and serve as a template during the surgery. Resins created especially for use in medical applications are guaranteed to be safely used in operating theatre. Unfortunately extremely high costs for hardware and material limits the routine use of this technique in maxillofacial surgery.

Mcor Technologies Matrix 300 printer, which was already validated for clinical applications $[22,23]$ allows to produce low-cost and durable paper-based 3D models. Contrary to other commercial solutions it was not designed to be used in medicine, but for design and architecture. Therefore, in order to safely use 3D paperbased models in operating theatre, we need to check if these models could be sterilized and if these 3D models present with any cell cytotoxicity.

\section{Materials and methods}

In this study Matrix 300 (MCor Technologies, Dunleer, Ireland) paper based 3D printer was used. It uses $80 \mathrm{gsm}$ sheets of A4 paper and water soluble adhesive (MCor Technologies, Dunleer, Ireland) to produce detailed prints. The resolution of printing is $0,01 \mathrm{~mm}$. Printing process begins in PC running software controlling Matrix 300 printer called SliceIT (MCor Technologies, Dunleer, Ireland). This software allows creating or importing of earlier prepared stl file models and transforms them into data necessary for the Matrix 300 printer. Every object is analysed and cut into $0,1 \mathrm{~mm}$ layers equal to the thickness of the used A4 sheet. Such prepared 2D data are send to 3D printer where a cutting tungsten blade cut the object, layer by layer, from paper sheets. The layers are glued with Mcor Technologies Adhesive. When the 
printing process is finished the printed objects are freed from the waste in a process called "weeding". In this stage the excess paper from around the printed 3D object is removed.

In order to check the possibility of safe intra-operative use of 3D models from MCor Technologies Matrix 300 printer we tested the influence of sterilization on 3D printed objects shape stability and we determined the 3D models cytotoxicity. To determine cytotoxicity XTT test and calcein/ethidium dyeing were performed by Nanostructural and Molecular Biophysics Laboratory in Technopark Lodz. Comparison of adhesive to a normal glue for wood and paper was also performed. For comparison, normal glue for paper/wood adhesion (joiner glue, Wytwornia Chemiczna Dragon, Krakow, Poland) was used for investigation. Lenticular shape samples were prepared in polyethylene mini dishes and later their macro and microstructure was compared i.e. scanning electron microscope (SEM) images were compared (Figure 1).

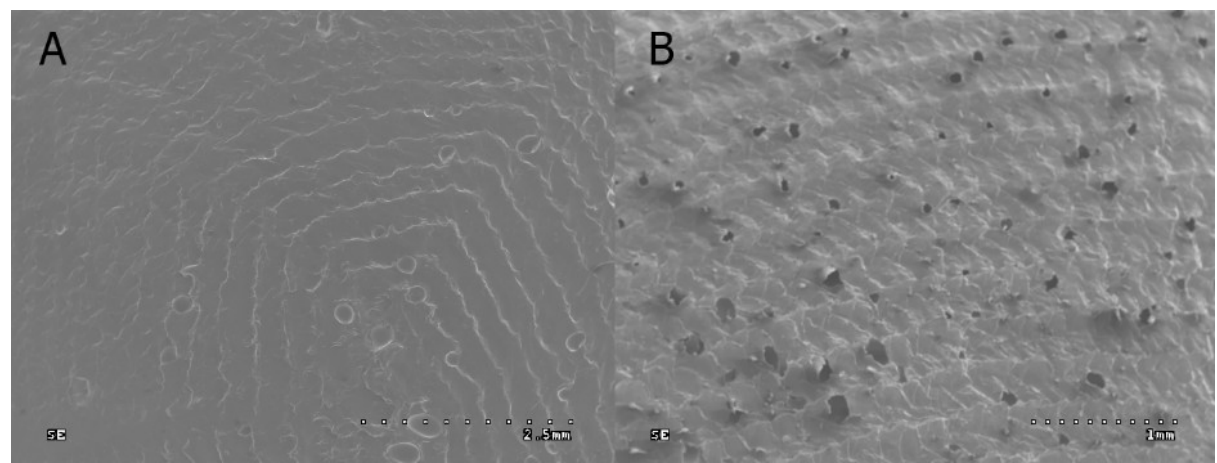

Fig. 1. Comparison of scanning electron microscope images of glue samples after drying. A. Surface of MCor adhesive; B. Surface of normal glue for paper/wood adhesion.

Also the spectral analysis (Table 1) of analyzed adhesives was performed with Thermo Noran system (Thermoscientific, Waltham, USA). 
Table 2. Cuboids dimensions related to sterilization methods

\begin{tabular}{|l|l|l|l|l|}
\hline $\begin{array}{l}\text { Sterilization } \\
\text { method }\end{array}$ & Dimension & $\begin{array}{l}\text { Before } \\
\text { sterilization } \\
\text { [mm] }\end{array}$ & $\begin{array}{l}\text { After } \\
\text { sterilization } \\
\text { [mm] }\end{array}$ & $\begin{array}{l}\text { Statistical } \\
\text { significance } \\
\text { (p) }\end{array}$ \\
\hline radiation & $\mathrm{x}$ & 9.95 & 9.93 & 0.121 \\
\hline radiation & $\mathrm{y}$ & 30.15 & 30.15 & 0.509 \\
\hline radiation & $\mathrm{z}$ & 20.13 & 20.13 & 0.096 \\
\hline gas plasma & $\mathrm{x}$ & 9.90 & 9.90 & 0.792 \\
\hline gas plasma & $\mathrm{y}$ & 30.19 & 30.19 & 0.434 \\
\hline gas plasma & $\mathrm{z}$ & 20.15 & 20.16 & 0.505 \\
\hline ethylene oxide & $\mathrm{x}$ & 9.90 & 9.91 & 0.066 \\
\hline ethylene oxide & $\mathrm{y}$ & 30.22 & 30.21 & 0.309 \\
\hline ethylene oxide & $\mathrm{z}$ & 20.16 & 20.17 & 0.053 \\
\hline
\end{tabular}

Table 1. Spectral analysis of Mcor Technologies adhesive and normal wood/paper glue (Dragon). Comparison of percentage of number of atoms creating adhesive molecules.

\begin{tabular}{|l|l|l|}
\hline & Normal glue & $\begin{array}{l}\text { Mcor Technologies } \\
\text { adhesive }\end{array}$ \\
\hline Carbon & $62,86 \%$ & $69,64 \%$ \\
\hline Oxygen & $36,64 \%$ & $30,36 \%$ \\
\hline Calcium & $0,50 \%$ & - \\
\hline
\end{tabular}

In order to verify the influence of sterilization on the shape stability we printed 30 cuboids with dimensions $10 \times 20 \times 30 \mathrm{~mm}$. The cuboids were divided into three groups by ten samples at random. For each group we tested one of current procedures of low temperature sterilization used in hospitals: 1 . Ethylene oxide in temperature of $55^{\circ} \mathrm{C}$, with a time of 4.5 hours and degazation period of 12 hours; Hydrogen peroxide gas plasma in temperature of $60^{\circ} \mathrm{C}$; and Gamma irradiation at $21^{\circ} \mathrm{C}$ with $25 \mathrm{kGy}$. Each cuboid was measured by observer using a calliper three times before and three times after sterilization (Table 2). Results were analysed statistically in Statgraphics Plus (Summary Statistics, ANOVA, analysis of linear regression, t-test). Statistical significance was determined as $\mathrm{p}<0.05$. 
Before sterilization [mm] - mean dimension before sterilization [mm]

After sterilization [mm] - mean dimension after sterilization [mm]

Statistical significance (p) - statistical significance value calculated with t-test statistics. For each $p$ value higher than 0,05 there is no statistically important change in dimension before and after sterilization.

In order to verify cytotoxicity of 3D prints from MCor Technologies sixty cylinders with a high of $3 \mathrm{~mm}$ and diameter of $14 \mathrm{~mm}$ were printed. They were divided into six groups with ten samples in each group. Three of those groups were covered before sterilization with 2-octyl-cyanoacrylate (Dermabond topical skin adhesive by Ethicon LLC, San Lorenzo, Puerto Rico) (Figure 2).

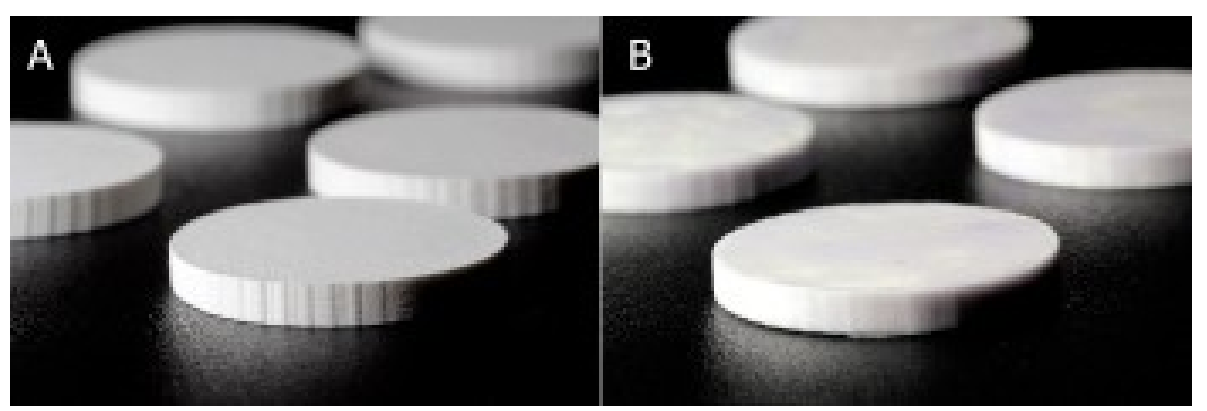

Fig. 2. 3D printed cylinders used for cytotoxicity determination tests. A. Before coating with 2-octyl-cyanoacrylate; B. After coating with 2-octylcyanoacrylate (Dermabond topical skin adhesive by Ethicon LLC, San Lorenzo, Puerto Rico).

The test cylinders were covered by 2-octyl-cyanoacrylate in the following pattern: the wall first, after five minutes the base and after further five minutes the second base. The same procedure was performed once again after ten minutes. Three different methods of sterilization (radiation, plasma, ethylene oxide) were used. Samples after sterilization were used for further cytotoxicity analysis:

Intact + ethylene oxide sterilization -10 pieces

Coated + ethylene oxide sterilization -10 pieces

Intact + gas plasma sterilization -10 pieces

Coated + gas plasma sterilization -10 pieces

Intact + radiation sterilization - 10 pieces

Coated + radiation sterilization -10 pieces

Additionally there were two control samples:

Control (+): intact medium and culture of XXT

Control (-): intact medium and culture washed with 50\% ethanol. 
The cytotoxicity of 3D printed paper blocks was tested by Nanostructural and Molecular Biophysics Laboratory in Technopark Lodz using normal adult human dermal fibroblasts (ATCC No. PCS-201-012, ATCC, Manassas, USA). Survival of cells was tested using spectrophotometry with XTT and was defined as ratio of absorbency of tested probe to absorbency of control probe.

\section{Cell culture preparation}

Normal, human, adult dermal fibroblast (ATCC No. PCS-201-012, ATCC, Manassas, USA) culture was grown in $75 \mathrm{~cm} 2$ tissue culture dish (MIDSCI Company, St. Louis, USA) using classical method of single layer cell culture until the phase of late logarithmic growth. Cells after moving from temperature $-150^{\circ} \mathrm{C}$ and unfreezing were put in a Fibroblast Basal Medium (ATCC No. PCS-201-030, ATCC, Manassas, USA) enriched with Fibroblast Growth Kit - Low Serum (ATCC No. PCS-201041, ATCC, Manassas, USA) and antibiotics Penicillin and Streptomycin (Sigma No. P0781, Sigma-Aldrich, St. Louis, USA). Cultures were grown in an incubator at $37^{\circ} \mathrm{C}, 5 \% \mathrm{CO}_{2}, 19 \% \mathrm{O}_{2}$ and humidity $100 \%$. After reaching confluence of $80 \%$ cell cultures were washed with Dulbecco`s Phosphate Buffered Saline (DPBS) (BI No. 02-023-1A, Biological Industries, Kibbutz Beit Haemek, Israel). In order to break cells connections $0.6 \mathrm{ml}$ of trypsin/EDTA (Sigma No. T3924, Sigma-Aldrich, St. Louis, USA) was used. Trypsinization was performed in incubator for five minutes. The process was ended by diluting trypsin with growth medium five times. Cells after freeing them from single layer culture were suspended in growth medium and were counted with Thoma chamber. Dead cells were marked with $0.4 \%$ trypan blue.

\section{Preparation of samples}

Discs of analysed substance were placed in an aseptic 6-well tissue culture plate and were flooded with $3 \mathrm{ml}$ of complete Fibroblast Basal Medium. After 24 hours medium containing substances unbound from analysed discs was added to cell cultures. Negative control were cells treated with $50 \%$ ethanol. Method was taken from norm PN-EN-ISO 10993-12 [24].

\section{Cytotoxicity analysis using spectrophotometric method with XTT}

The XTT method uses the ability of mitochondrial dehydrogenases, especially succinic to transform tetrazolic salt of XTT to formazan product. This reaction is only possible in metabolically active cells. Cells in number $4 \times 10^{3}$ cells $/ \mathrm{ml} /$ well were placed in an aseptic 96 well flat-bottomed spectrophotometric plate and were cultured for 24 hours (minimal time required for cells attachment to the cell culture 
plate). After this time conditioned medium was removed from the culture and was replaced with $0.1 \mathrm{ml}$ medium containing substance unbound form analysed discs. After another 24 hours of incubation medium containing unbound substances was removed by aspiration, culture was washed with DPBS and $50 \mu \mathrm{l} /$ well XTT mixture (BI No. 20-300-1000, Biological Industries, Kibbutz Beit Haemek, Israel) was added. Cultures with XTT were incubated for 4 hours in $37^{\circ} \mathrm{C}$. Absorbance of formozan solution was read on Multiskan GO (Thermoscientific, Waltham, USA) plate reader for wave length $450 \mathrm{~nm}$ were reference wave length was $630 \mathrm{~nm}$. Survival rate of cells was established from the relation: Survival $(\%)=(\mathrm{A} / \mathrm{Ak}) \times 100 \%$, where A is absorbance of sample treated with analysed substance, AK is absorbance of control sample (not treated with analysed substances).

\section{Calcein/Ethidium dyeing}

Calcein AM is commonly used to mark the living cells due to its ability to freely transfer through the cell membrane. In the cell AM (acetometoxy) group is degraded enzymatically which results in attaching calcium ions to the particle causing high fluorescence of the probe. Ethidium homodimer enters cells with damaged membranes and binds to nucleic acids, thereby dyeing dead cells.

Discs of analysed substance were placed in an aseptic 6-well tissue culture plate and were seeded with cells in number $8 \times 10^{4}$ cells $/ \mathrm{ml} /$ well in $3 \mathrm{ml}$ growth medium. After $24 \mathrm{~h}$ incubation cultures were washed twice with DPBS and $3 \mathrm{ml}$ of fluorescent dyes calcein/ethidium in DPBS were added according to LIVE/DEAD Viability/Cytotoxicity Kit (Molecular Probes No. L3224, , Life Technologies, Waltham, USA) protocol. After 30 minutes of incubation samples were washed with DPBS. Observation was done under GX71 (Olympus, Tokyo, Japan) fluorescence microscope.

\section{Results}

MCor Adhesive specification is not available from the producer (MCor Technologies, Dunleer, Ireland). The appearance of the MCor adhesive is very similar to a normal glue for paper/wood adhesion i.e. cream, white opaque, and typical smell. Lenticular shape samples were prepared in polyethylene mini dishes. The viscosity of MCor adhesive is lighter than joiner glue. That is why the surface of MCor sample was just glossy meniscus, and in fresh joiner glue the surface was wrinkled. After 24 hours of adhesives hardening, it was noticed significant surface deformation in joiner glue. The surface formed concavity contrary to near flat surface of MCor glue. Anyway the shrinkage was also observed in MCor adhesive. In Spectral analysis occurred that both adhesives are comprised mostly of carbon and oxygen. However, in the normal glue small amount of calcium was also found (Table 1). SEM 
analysis of samples showed smoother surface of MCor Adhesive compared to normal paper/wood glue (Figure 1).

There was no statistically significant difference for established statistical significance $\mathrm{p}=0.05$ in cuboids dimensions before and after sterilization regardless of sterilization method (Table 2).

In XTT analysis samples showed higher cytotoxicity against normal, human, adult dermal fibroblast culture when compared to positive control. However, statistical analysis of gathered results showed that nearly all analysed samples showed statistically lower cytotoxicity than negative control. The only exception was group sterilised in ethylene oxide without Dermabond coating. In this group survival rate of cells (average 17.24\%) was similar to negative control group (average 16.65\%). The ANOVA statistical analysis confirmed that 2-octyl cyanoacrylate coating (Figure 2) of paper model improved biological behaviour of the material. It decreased cytotoxicity of the model independently of sterilization method (Table 3).

Table 3. Absorbance rates and cell survival rate depending on sterilisation method. Survival $(\%)=(A / A k) \times 100 \%$ where $A$ is absorbance of sample treated with analysed substance, and Ak is absorbance of control sample.

\begin{tabular}{|l|l|l|}
\hline & Mean absorbance of sample & Mean survival rate (\%) \\
\hline Control $(+)$ & 0.224309091 & $100.00 \%$ \\
\hline Control $(-)$ & 0.037354545 & $16.65 \%$ \\
\hline EO INTACT & 0.038663636 & $17.24 \%$ \\
\hline EO COATED & 0,046381818 & $20.68 \%$ \\
\hline GP INTACT & 0.042927273 & $19.14 \%$ \\
\hline GP COATED & 0.0838 & $37.36 \%$ \\
\hline IR INTACT & 0.053254545 & $23.74 \%$ \\
\hline IR COATED & 0.070872727 & $31.60 \%$ \\
\hline
\end{tabular}

Control (+) - intact medium and culture of XXT

Control (-) - intact medium and culture washed with $50 \%$ ethanol

EO INTACT - intact samples (not covered with dermabond cyanoacrylate) + ethylene oxide sterilization

EO COATED - samples covered with dermabond cyanoacrylate + ethylene oxide sterilization

GP INTACT - intact samples (not covered with dermabond cyanoacrylate) + gas plasma sterilization

GP COATED - samples covered with dermabond cyanoacrylate + gas plasma sterilization 
IR INTACT - intact samples (not covered with dermabond cyanoacrylate) + radiation sterilization

IR COATED - samples covered with dermabond cyanoacrylate + radiation sterilization.

In calcein/ethidium dyeing test due to the high fluorescence of the background caused by cylinders of analysed substance it was impossible to perform exact analysis of number of marked cells. Samples not covered with 2-octyl cyanoacrylate strongly absorbed growth medium what caused increase in volume and stratification of samples.

\section{Discussion}

No dimensional change and no stratification after sterilization were the minimal requirements for $3 \mathrm{D}$ models that were planned to be used in the operating theatre. Three different, and current methods of sterilization were chosen for this study. None of those methods had any significant impact on size and structure of printed models. However, sterilization with gas plasma was delayed by the sorption of hydrogen superoxide by printed cuboids. This may result in failure of sterilization in case of bigger models.

In order to be safely used as a surgical template printed model should have as low cytotoxic effect as possible. Three-dimensional prints from Mcor Technology Matrix 300 paper-based printer had shown significant cytotoxic effect. Although cyanoacrylate adhesives are discussed as being cytotoxic themselves [25], coating the models with Dermabond cyanoacrylate tissue adhesive significantly reduced cytotoxicity. What is more, samples covered with Dermabond did not absorbed growth medium in calcein/ethidium dyeing test and showed no stratification effect at all in comparison to intact samples. Further studies regarding safe covering of $3 \mathrm{D}$ printed models are required. 
- Acknowledgements: this study was presented at the Congress of European Association of cranio-maxillofacial surgery, Prague, Czech Republic, 2014.

- Funding sources statement: this study does not receive any funding.

- Competing interests: Olszewski R is Editor-in-chief of Nemesis. The other authors have no competing interests related to this study. Compliance with ethical standards

- Ethical approval: there was no need for ethical committee approval for this experimental study.

- Informed consent: there was no need for informed consent for this experimental study.

Authors contribution:

\begin{tabular}{|c|c|}
\hline Author & Contributor role \\
\hline Kozakiewicz M & $\begin{array}{l}\text { Conceptualization, Data curation, } \\
\text { Investigation, Methodology, Validation, } \\
\text { Resources, Writing original draft } \\
\text { preparation, Writing-review and editing }\end{array}$ \\
\hline Szymor P & $\begin{array}{l}\text { Conceptualization, Data curation, } \\
\text { Investigation, Methodology, Validation, } \\
\text { Writing original draft preparation, Writing- } \\
\text { review and editing }\end{array}$ \\
\hline Olszewski R & $\begin{array}{l}\text { Resources, Validation, Writing original } \\
\text { draft preparation, Supervision, Writing } \\
\text { original draft preparation, Writing-review } \\
\text { and editing }\end{array}$ \\
\hline
\end{tabular}

\section{References}

1. Wilde F, Winter K, Kletsch K, Lorenz K, Schramm A. Mandible reconstruction using patient-specific pre-bent reconstruction plates: comparison of standard and transfer key methods. Int J Comput Assist Radiol Surg 2015;10:129-140.

2. Kozakiewicz M, Szymor P (2013) Comparison of pre-bent titanium mesh versus polyethylene implants in patient specific orbital reconstructions. Head Face Med 9:32.

3. Essig H, Dressel L, Rana MM, Kokemueller H, Ruecker M, Gellrich NC. Precision of posttraumatic primary orbital reconstruction using individually bent titanium mesh with and without navigation: a retrospective study. Head Face Med 2013;9:18. 
4. Kozakiewicz M, Elgalal M, Loba P, Komuński P, Arkuszewski P, BroniarczykLoba A, Stefańczyk L. Clinical application of 3D pre-bent titanium implants for orbital floor fractures. J Craniomaxillofac Surg 2009;37:229-234.

5. Metzger MC, Schön R, Weyer N, Rafii A, Gellrich NC, Schmelzeisen R, Strong BE. Anatomical 3-dimensional pre-bent titanium implant for orbital floor fractures. Ophthalmology 2006;113:1863-1868.

6. Salmi M, Paloheimo KS, Tuomi J, Wolff J, Mäkitie A. Accuracy of medical models made by additive manufacturing (rapid manufacturing). J Craniomaxillofac Surg 2013;41:603-609.

7. Essig H, Rana M, Kokemueller H, von See C, Ruecker M, Tavassol F, Gellrich NC. Pre-operative planning for mandibular reconstruction - a full digital planning workflow resulting in a patient specific reconstruction. Head Neck Oncol 2011;3:45.

8. Mertens C, Löwenheim H, Hoffmann J. Image data based reconstruction of the midface using a patient-specific implant in combination with a vascularized osteomyocutaneous scapular flap. J Craniomaxillofac Surg 2013;41:219-225.

9. Olszewski R, Reychler H. Three-dimensional surgical guide for frontal-nasalethmoid-vomer disjunction in Le Fort III osteotomy. J Craniofac Surg 2011;22:1791-1792.

10. Seres L, Varga E, Kocsis A, Rasko Z, Bago B, Varga E, Piffko J. Correction of a severe facial asymmetry with computerized planning and with the use of a rapid prototyped surgical template: a case report/technique article. Head Face Med 2014;10:27.

11. Stoetzer M, Rana M, von See C, Eckardt AM, Gellrich NC. Reconstruction of defects of maxillary sinus wall after removal of a huge odontogenic lesion using prebended 3D titanium-mesh and CAD/CAM technique. Head Face Med 2011;7:21.

12. Chang PS, Parker TH, Patrick CW, Miller MJ. The accuracy of stereolithography in planning craniofacial bone replacement. J Craniofac Surg 2003;14:164-170.

13. Kozakiewicz M. Computer-aided orbital wall defects treatment by individual design ultrahigh molecular weight polyethylene implants. J Craniomaxillofac Surg 2014;42:283-289.

14. Olszewski R (2013) Three-dimensional rapid prototyping models in craniomaxillofacial surgery: systematic review and new clinical applications. Proc Belgian R Acad Med 2:43-77. 
15. Ibrahim D, Broilo TL, Heitz C, de Oliveira MG, de Oliveira HW, Nobre SM, Dos Santos Filho JH, Silva DN. Dimensional error of selective laser sintering, three-dimensional printing and PolyJet models in the reproduction of mandibular anatomy. J Craniomaxillofac Surg 2009;37:167-173.

16. Silva DN, Gerhardt de Oliveira M, Meurer E, Lopes da Silva JV, Santa-Bárbara A. Dimensional error in selective laser sintering and 3D-printing of models for craniomaxillary anatomy reconstruction. J Craniomaxillofac Surg 2008;36:443449.

17. Rohner D, Guijarro-Martínez R, Bucher P, Hammer B. Importance of patientspecific intraoperative guides in complex maxillofacial reconstruction. J Craniomaxillofac Surg 2013;41:382-390.

18. Esses SJ, Berman P, Bloom AI, Sosna J. Clinical applications of physical 3D models derived from MDCT data and created by rapid prototyping. AJR Am J Roentgenol 2011;196:W683-688.

19. Rengier F, Mehndiratta A, von Tengg-Kobligk H, Zechmann CM, Unterhinninghofen R, Kauczor HU, Giesel FL. 3D printing based on imaging data: review of medical applications. Int J Comput Assist Radiol Surg 2010;5:335-341.

20. Choi JY, Choi JH, Kim NK, Kim Y, Lee JK, Kim MK, Lee JH, Kim MJ. Analysis of errors in medical rapid prototyping models. Int J Oral Maxillofac Surg 2002;31:23-32.

21. Murugesan K, Anandapandian PA, Sharma SK, Vasantha Kumar M. Comparative evaluation of dimension and surface detail accuracy of models produced by three different rapid prototype techniques. J Indian Prosthodont Soc 2012;12:16-20.

22. Olszewski R, Szymor P, Kozakiewicz M (2014) Accuracy of three-dimensional, paper-based models generated using a low-cost, three-dimensional printer. J Cranio-Maxillofacial Surg 2014;42:1847-1852.

23. Szymor P, Kozakiewicz M, Olszewski R. Accuracy of open-source software segmentation and paper-based printed three-dimensional models. J Craniomaxillofac Surg 2016;44:202-209.

24. Biological evaluation of medical devices. Part 12: Sample preparation and reference materials. PN-EN-ISO 10993-12 2012.

25. Thumwanit V, Kedjarune U. Cytotoxicity of polymerized commercial cyanoacrylate adhesive on cultured human oral fibroblasts. Aust Dent J 1999;44:248-252. 
a three-dimensional paper-based printer 